\title{
High Power THz Generation from Sub-ps Bunches of Relativistic Electrons
}

\author{
S. Benson, D. R. Douglas, H. F. Dylla, J. Gubeli, K. Jordan, G. R. Neil, M. Shinn, S. Zhang and \\ G.P. Williams \\ FEL Facility, Jefferson Lab, 12000 Jefferson Avenue, Newport News, VA 23606
}

\begin{abstract}
We describe a $>100$ Watt broadband $\mathrm{THz}$ source that takes advantage of the relativistic enhancement of the radiation from accelerating electrons according to the formula assigned the name of Sir Joseph Larmor[1,2]. This is in contrast to the typical 1 milliwatt sources available in a laboratory. Specifically, for relativistic electrons the emission is enhanced by the fourth power of the increase in mass. Thus for $100 \mathrm{MeV}$ electrons, for which the mass increases by a factor of $\sim 200$, the enhancement is $>10^{9}$. The experiments use a new generation of light source called an energy recovery linac (ERL) [3], in which bunches of electrons circulate once, but in which their energy is recovered. In such a machine the electron bunches can be very much shorter than those, say, in storage rings or synchrotrons.

The Jefferson Lab facility operates in new limits of emission from relativistic particles involving both multiparticle coherence and near-field emission in which the velocity (Coulomb) term in the classical electrodynamical theory becomes as important as the acceleration term (synchrotron radiation).

The sub-picosecond pulses of light offer unique capabilities in 2 specific areas, namely timeresolved dynamics, and imaging. High resolution $\mathrm{THz}$ spectroscopy has recently revealed sharp vibrational modes for many materials including malignant tissue, proteins, DNA, pharmaceuticals and explosive materials. Energetically the $\mathrm{THz}$ range embraces superconducting bandgaps, and regions of intense interest in the understanding of systems in which correlated motions of electrons are important, such as colossal magneto-resistive and high-Tc materials. The very high power levels of the new source will allow non-linear effects to be observed as well as the creation of novel states of materials, including electric-field driven localization[4]. We will give examples of existing work in these areas and present opportunities afforded by the new source.
\end{abstract}

\section{INTRODUCTION}

In this paper we compare and contrast a new high power $\mathrm{THz}$ source with conventional Auston switch[5] sources, and synchrotron radiation sources. We also discuss in detail the design of our user facility, and in particular the beam transport system that feeds it. We point out that this facility can probe materials at frontiers of ultra-fast and ultra-high electric and magnetic fields, and in addition can provide multiple photon beams for 2 or 3 photon pump-probe experiments such as those described above. 


\section{PHYSICS OF LIGHT PRODUCTION FROM ACCELERATED ELECTRONS - COMPARISON WITH STANDARD SOURCES}

Propagating fields of light can be generated from Maxwell's equation:

$$
\nabla \times H=J_{\text {free }}+\varepsilon \frac{\partial E}{\partial t}+\frac{\partial P}{\partial t}
$$

which shows how a free current $J$ can generate propagating electric $(E)$ and magnetic $(H)$ fields, and is the basis of most radio, television and cellular telephone transmission systems.

From this equation with relativistic corrections one can obtain Larmor's formula which gives the radiated power per electron:

$$
\text { Radiated Power }=\frac{3 e^{2} a^{2}}{c^{3}} \gamma^{4}
$$

where e is the charge on an electron, a the acceleration, $\mathrm{c}$ the velocity of light, and gamma the ratio of the mass of the electron ratioed to the rest mass.

In the next 2 paragraphs we compare the essential physics of our $\mathrm{THz}$ source with that of a more standard source.

In a conventional $\mathrm{THz}$ source, namely that based on an Auston switch, a laser excites charge carriers in a semiconductor and they are accelerated in a large field, usually of the order of 100 volts across 100 microns. Importantly, though, the laser is pulsed in the sub-ps time range so that the charge carriers all contribute in-phase to the generated field, so that the power scales as the number of carriers squared. This is called super-radiance. It is important to note, however, that gamma, in Eq. (2), is unity.

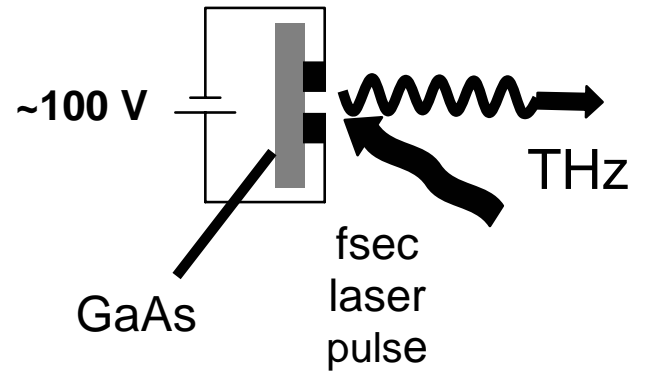

$$
\begin{gathered}
E=\frac{100 \mathrm{~V}}{10^{-4} \mathrm{~m}}=10^{6} \mathrm{~V} / \mathrm{m} \\
a=\frac{F}{m}=\frac{10^{6} \mathrm{~V} \cdot e / \mathrm{m}}{.5 \mathrm{MeV} / \mathrm{c}^{2}}=\frac{10^{6}\left(3 \times 10^{8}\right)^{2}}{0.5 \times 10^{6}} \\
\cong 10^{17} \mathrm{~m} / \mathrm{sec}^{2}
\end{gathered}
$$

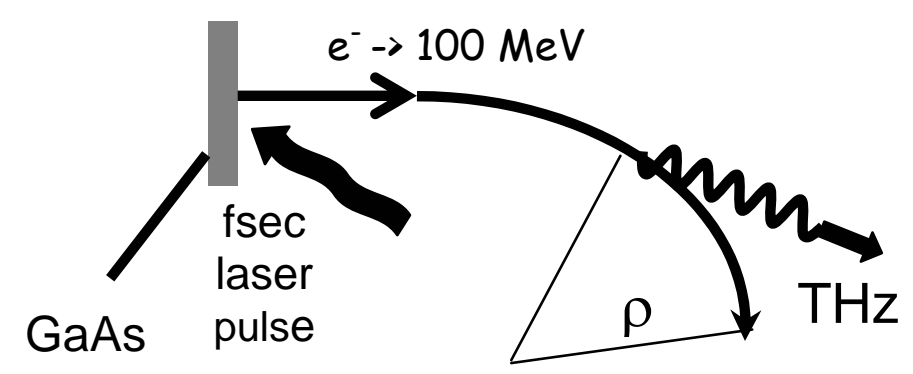

$$
\begin{gathered}
a=\frac{c^{2}}{\rho}=\frac{\left(3 \times 10^{8}\right)^{2}}{1} \cong 10^{17} \mathrm{~m} / \mathrm{sec}^{2} \\
\text { if } \rho=1 \mathrm{~m}
\end{gathered}
$$

Fig. 1. Comparison between a conventional $\mathrm{THz}$ source based on an Auston switch, left, and the relativistic source described here on the right. The acceleration and number of carriers is approximately the same in both cases. 
In our $\mathrm{THz}$ source we exploit the enhancement brought about by increasing gamma, otherwise the generation of $\mathrm{THz}$ light is similar in following Eq. 2. We begin with a sub-picosecond laser that produces carriers in a semiconductor. However in our case these carriers (or at least the electrons) are accelerated out of the semiconductor into free space where they are further accelerated to $\sim 100 \mathrm{MeV}$. Note that the rest mass of an electron is $0.5 \mathrm{MeV}$ so gamma is now 200 , and the enhancement will be $200^{4}$, or $\sim 9$ orders of magnitude!! In practice the hundreds of microwatts from the Auston switch are converted into kilowatts at the JLab source. The discrepancy is almost certainly due to the differences in the number of participating carriers, since the physics is exact.

There is another advantage of the relativistic source, which concerns the source emittance and thus brightness, or power per (area $\times$ angle). The area term is similar for both standard and relativistic sources, but the angle of emission of the relativistic source is Lorentz contracted[6], and is typically a solid angle of $10^{-2} \pi$ steradians instead of $2 \pi$, another factor of 100 gain when the JLab source is used for spectroscopy of small samples such as those in high pressure cells or high magnetic fields.

\section{THE JEFFERSON LAB HIGH POWER THZ FACILITY - COMPARISON WITH SYNCHROTRON LIGHT SOURCES.}

Although it is described in another talk in this meeting, we briefly describe the present accelerator facility on which the THz source is based. The JLab machine[3,7] is based on an energy recovered linac, or ERL, which comprises a closed loop structure in which energy, rather than electrons are stored. The energy is stored in the superconducting radio-frequency linac. Each bunch of electrons makes a single pass around the machine, and can thus be of subpicosecond length since there is insufficient time to come into longitudinal equilibrium as in storage rings. The charge per bunch in our machine is 135 picoCoulombs or $\sim 10^{9}$ electrons. In this machine, unlike the usual situation in storage rings, the bunch length is shorter than the wavelength being emitted, so that the electric fields of the different electrons are in phase, giving what is known as a multiparticle coherent enhancement. This is of the order of the number of electrons, namely $10^{9}$. Thus although the average current is only $10 \mathrm{~mA}$, compared to the hundreds of milliamps of most storage rings, it will emit as though the current is $10^{7}$ amps for wavelengths longer than the pulse.

There is, however, an additional feature of the JLab source that we exploit, and that is the electric field that is produced when the electron enters the magnetic field of the dipole. This is generated from a different Maxwell equation, namely:

$$
\nabla \times E=-\frac{\partial B}{\partial t}
$$

Such "edge-radiation" sources are being implemented at some synchrotron radiation facilities[8,9]. It turns out that in the situation pertaining to our geometry, our first mirror is in the near-field of the emitted light, and the edge radiation term is as large as the synchrotron radiation term. 


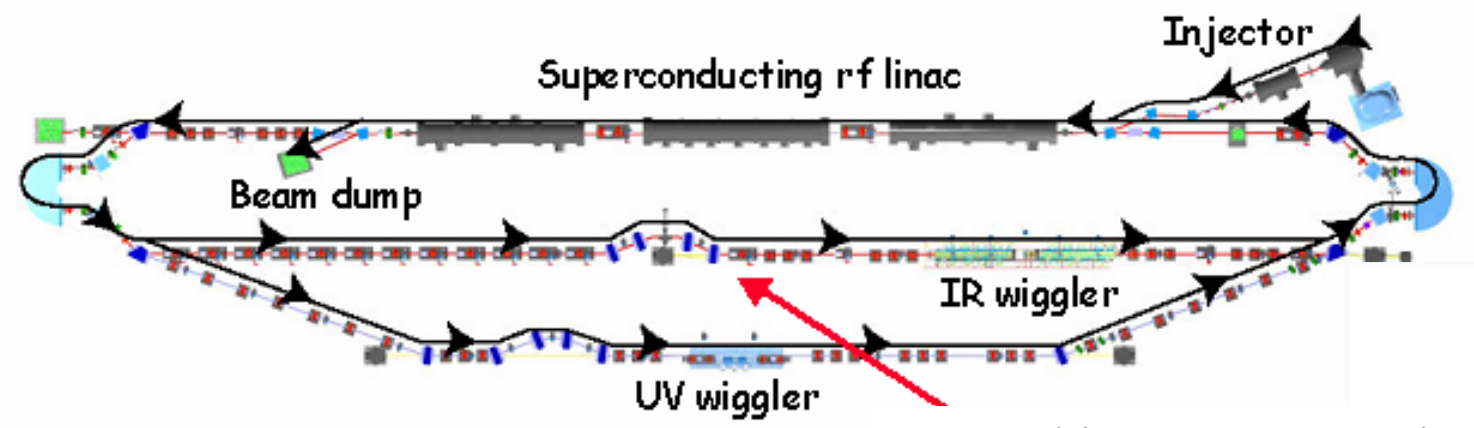

THz Chicane \& Extraction

Fig. 2. The Jefferson Lab Free Electron Laser showing the THz extraction location.

\section{THE THz BEAMLINE.}

In the JLab FEL the electron beam traverses a magnetic chicane to avoid the upstream mirror of the FEL cavity as shown in Fig. 2. This chicane also serves to compress the electron bunch, which has an energy chirp on it, so that the low energy electrons travel further in the magnetic field and are caught up by the higher energy ones at the tail. Thus this chicane provides the dipole field to generate the synchrotron radiation as well as the bunch compression that enhances the $\mathrm{THz}$ light emission. Into the chicane chamber we engineered a port that allows light to be collected into angles of 250 milliradians horizontally by 150 milliradians vertically.

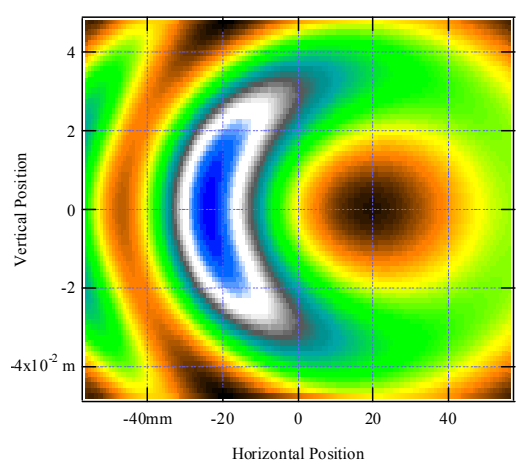

0.1 $\mathrm{THz}$

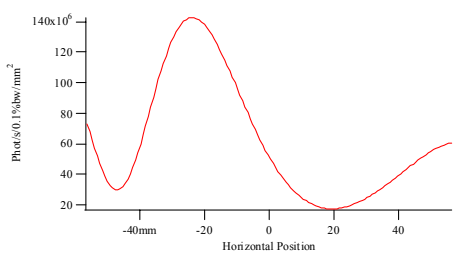

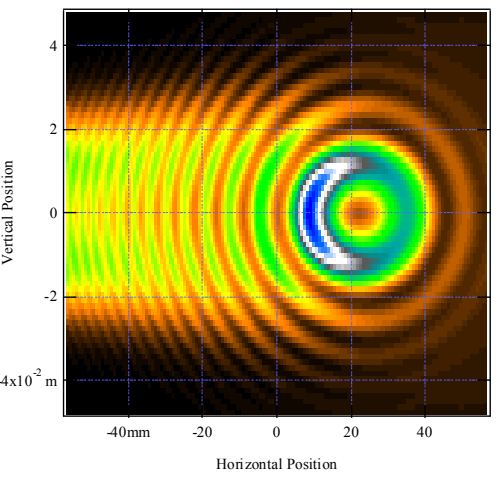

$1 \mathrm{THz}$

$33 \mathrm{~cm}^{-}$

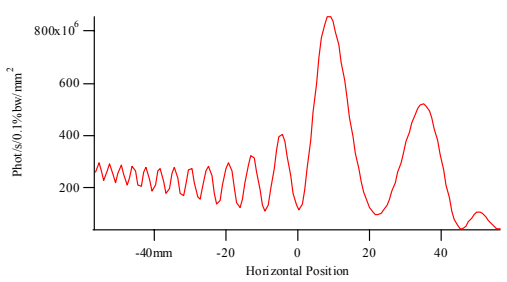

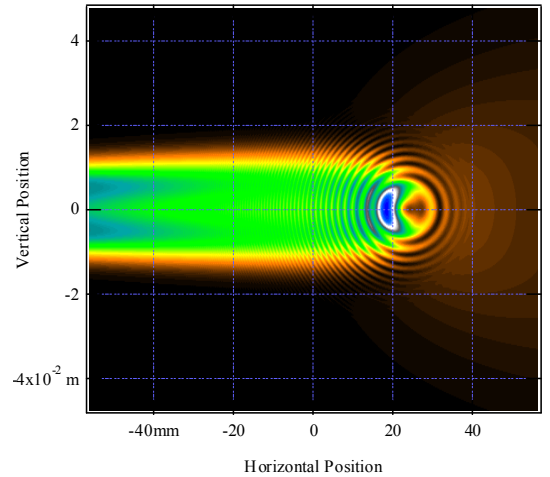

$10 \mathrm{THz}$ $330 \mathrm{~cm}^{-}$

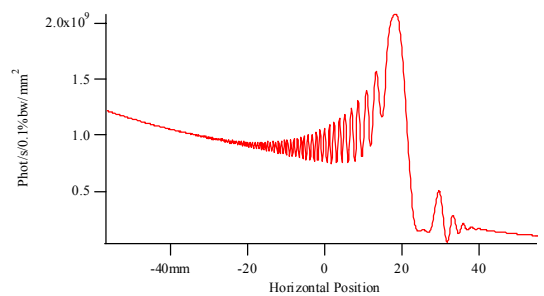

Fig. 3. Calculated patterns of light impinging on $\mathrm{M} 1$ for different frequencies, 2-D at the top, and horizontal cut at the bottom. The boxes at the top are approximately $100 \mathrm{~mm} \times 100 \mathrm{~mm}$. 
The light is collected by an ellipsoidal mirror (M1) that reflects it through an angle of $90^{\circ}$ and makes a $1: 1$ focus at a $20 \mathrm{~mm}$ diameter $1^{0}$ wedged diamond window. The focal length of this mirror is $625 \mathrm{~mm}$, and the mirror is fashioned out of uncoated solid aluminum. Due to the potential heat-loading, this mirror is cooled via a copper braid to a feedthrough. Due to the remote location of M1, it is mounted on a remotely controlled manipulator.

Patterns of light intensity generated by the accelerator are shown in Fig. 3. They were calculated using the Synchrotron Radiation Workshop (SRW) code developed and extended for our situation by Oleg Chubar[10]. The intensity patterns are dominated by the ring structure caused by interference from the upstream dipole edge. The circular structure comes from the edge radiation, while the build-up to the left of the horizontal cuts in the boxes at the bottom of Fig. 3 is due to the conventional synchrotron radiation. It can be seen that the angles into which the light is emitted vary considerably with photon energy, and that the present design collects most of the emission to $0.1 \mathrm{THz}$.

The portion of the beamline up to the diamond window is under machine vacuum, while the remainder of the beamline is at 100 millitorr.

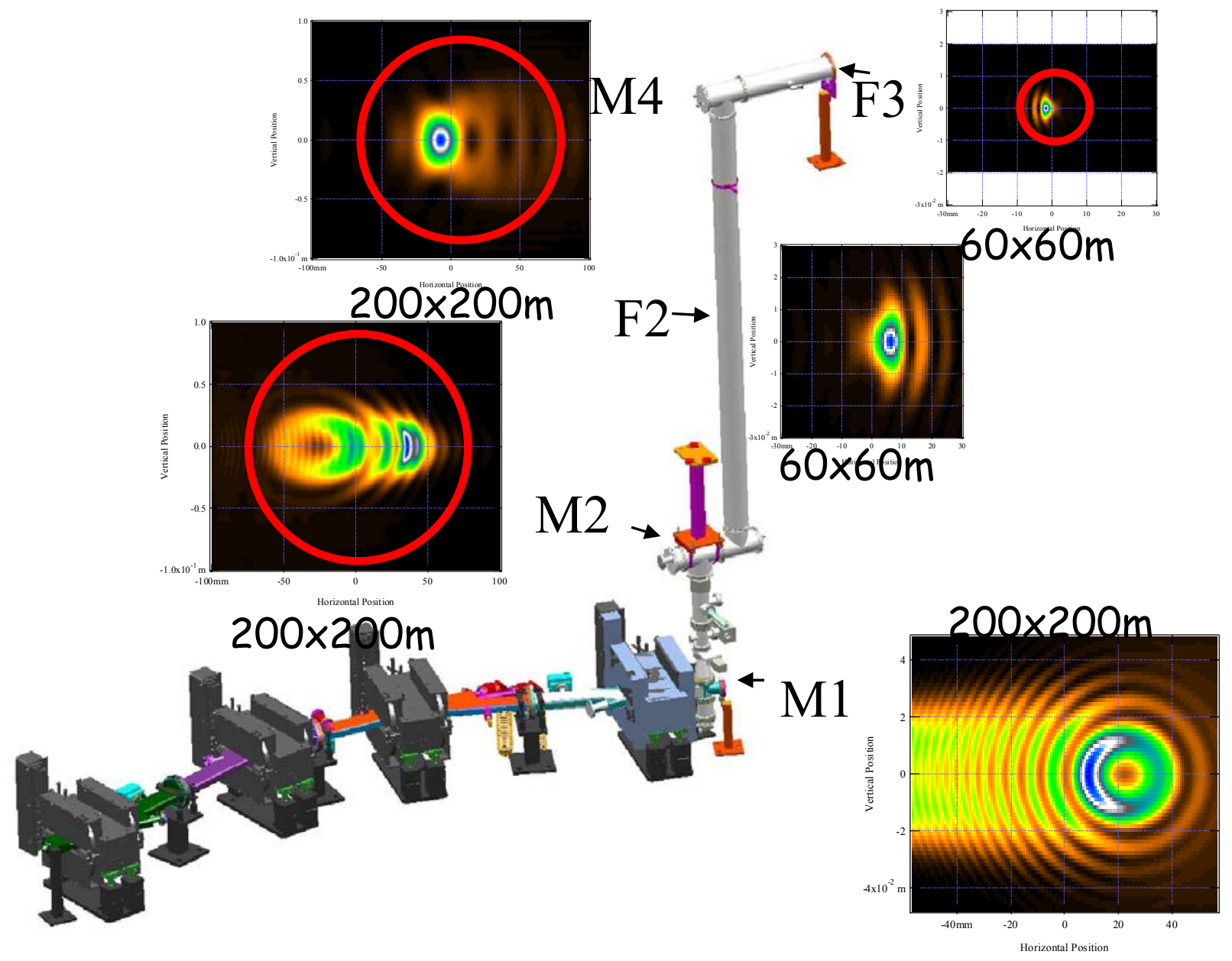

Fig. 4. 3-D rendering of the $\mathrm{THz}$ beamline at Jefferson Lab and patterns of light at some locations along the optical beam path. 
Light which exits the diamond window is intercepted by a second ellipsoidal mirror (M2) and deflected $372 \mathrm{~mm}$ parallel to the floor to a third plane mirror (M3) which deflects it vertically upwards. After passing a second focus at a distance of $2054 \mathrm{~mm}$ from M3, the beam impinges on M4 which is identical to M2, and which turns the beam horizontal in the laboratory, through a second diamond window if required, and onto an optical bench.

\section{THz LIGHT CHARACTERISTICS}

The intensity distributions shown in Fig. 4 were also propagated using the SRW code[10], and used to design the optics described above. They are shown in the beamline cartoon figure. The emitted light is primarily s-polarized, hence the mirror orientations. At the exit window the beam is $3 \mathrm{~mm}$ wide by $2 \mathrm{~mm}$ high and has a divergence of $\mathrm{f} / 8$. It is $60 \%$ polarized perpendicular to the floor of the laboratory, the polarization having been rotated by M4 deliberately so that it becomes s-polarized when it impinges on the beamsplitter in a standard Bruker $66 \mathrm{~V}$ spectrometer. The spectrum was also calculated and is shown in Fig. 5 for various bunch lengths. These calculations were made using a different code that takes account of the multiparticle coherent enhancement, and specifically the form factor of the longitudinal particle distribution within the electron bunch. Note also that the light is broadband and emitted in pulses at up to $75 \mathrm{MHz}$, each pulse containing up to 10 microjoules, depending on bunch length and charge.

Prior to constructing the present beamline we verified the calculations using an earlier incarnation of the JLab FEL. That machine was lower in energy and current, but produced 20 watts of broadband THz light in a spectrum that agreed well with our calculations, see Fig. 6.

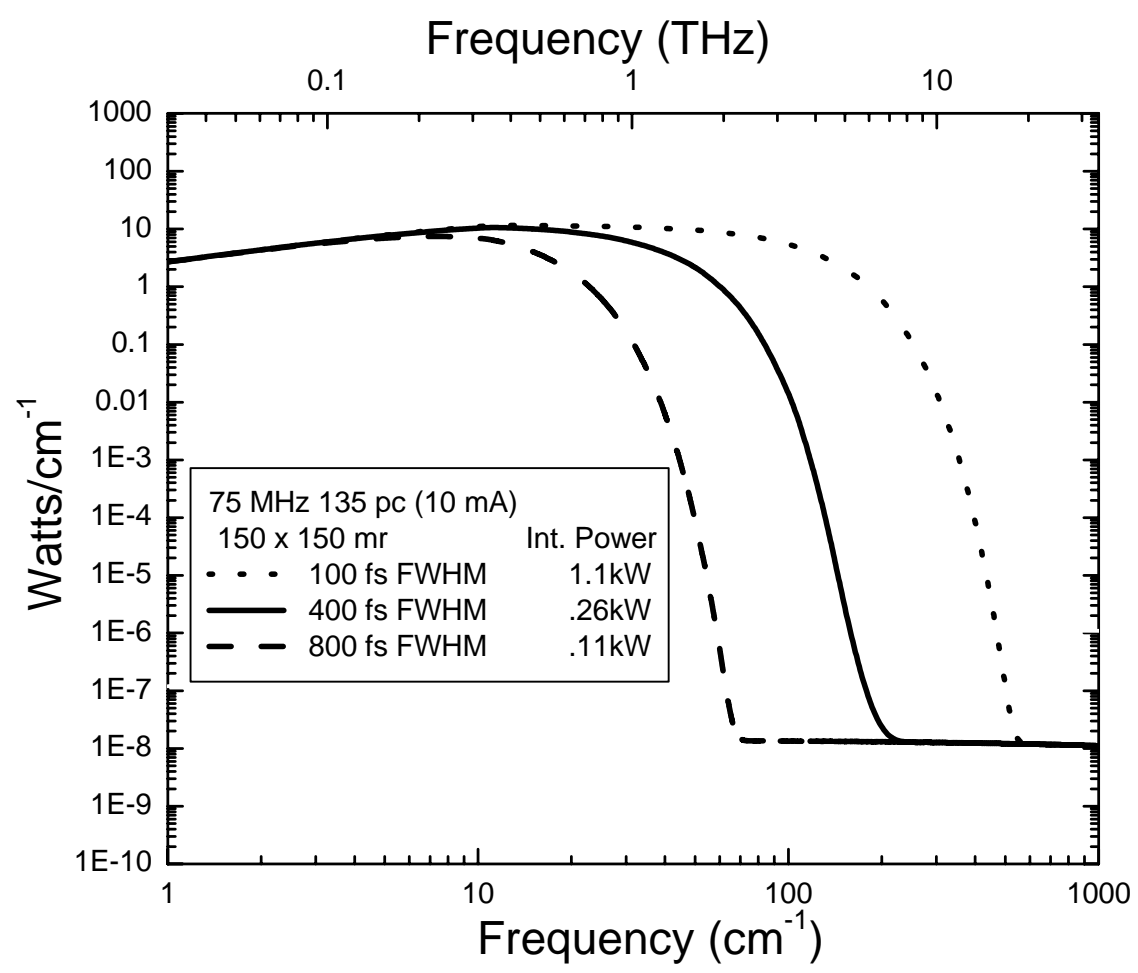

Fig. 5. Calculated average radiation power from the Jefferson Lab $\mathrm{THz}$ facility. 


\section{RESEARCH OPPORTUNITIES}

There are 4 areas of primary interest for research, large-scale imaging, spectroscopy, out-ofequilibrium dynamics, and non-linear behavior.

Imaging: At present with commercial Auston switch based systems, imaging is done by scanning, and it typically takes about 1 minute to image $1 \mathrm{~cm}^{2}$. These sources deliver about 500 microwatts into $2 \pi$ steradians about 100 microwatts of which can actually be collected and utilized. One advantage of such imaging systems is the coherent detection techniques that are typically used. With the present source, and with 100 watts available, all of which can be collected and utilized, large-scale imaging is possible assuming the availability of cameras. Using antenna-coupled bolometric arrays for example, whole body images $(50 \mathrm{~cm} \times 200 \mathrm{~cm})$ could be performed in seconds, since the area is $10^{3}$ times higher but the power is $10^{6}$ times higher.

Spectroscopy: Here the opportunity lies in areas where the sample is small or in environments of limited access or optical throughput, such as in high pressure cells or high magnetic fields. Now $1 \mathrm{~mA}$ of current in a synchrotron storage ring is equivalent in far-IR brightness to a $2000 \mathrm{~K}$ thermal source[11], and because our source has a current of $10 \mathrm{~mA}$ enhanced by the coherent emission from the $10^{9}$ electrons in the bunch, it therefore has an equivalent temperature of $10^{10} \mathrm{~K}$. Since in the far-IR, brightness is a function of T, then the brightness advantage is $10^{10}$.

Significantly, since work in the far-IR is usually governed by the $300 \mathrm{~K}$ background, the signal to noise is often the ratio between the lamp temperature and $300 \mathrm{~K}$. Thus the opportunities of

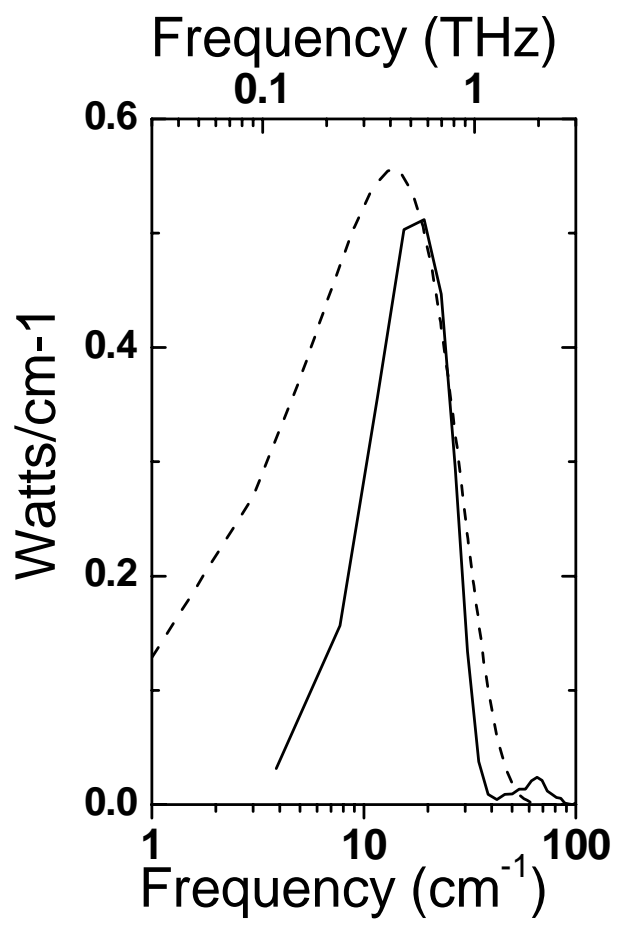

Fig. 6. Measured (solid) and calculated (dashed) spectra emitted from the relativistic electrons in the original Jefferson Lab FEL facility. The agreement of the onset and intensity is excellent, the discrepancy on the left hand side is due to reduced detection efficiency due both to diffraction and to the nature of the detector. 
utilizing a source with a high equivalent temperature will allow experiments to be performed at normal and even elevated temperatures for the first time.

Out-of-equilibrium dynamics: Here we are talking about using 2 synchronized photons to pump and then probe a system. One can imagine deriving the pump pulse from the same $\mathrm{THz}$ pulse as the probe, or utilizing the naturally synchronized FEL beam or another laser. Pioneering experiments in these areas have been carried out by Larry Carr at Brookhaven[12], and also by Gunter Luepke at our FEL facility[13].

Non-linear behavior: In these experiments one takes advantage of the high electric and concomitant magnetic fields that are present for a focused beam. Now the electric field generated by a certain power density is given by:

$$
E=\sqrt{\frac{W / m^{2}}{1.33 \times 10^{-3}}} \mathrm{~V} / \mathrm{m}
$$

Thus assuming 100 watts into a 500 micron diameter, and 300 fsec pulses every $10 \mathrm{nsec}$.

$$
\begin{aligned}
& \text { Area }=\pi\left(100 \times 10^{-6}\right)^{2} \mathrm{~m}^{2} \\
& \text { Peak Power }=100 \times \frac{10^{-8}}{300 \times 10^{-15}} \text { Watts } \\
& \text { Power / Area }=\frac{3 \times 10^{6}}{\pi\left(500 \times 10^{-6}\right)^{2}} \\
&=4 \times 10^{12} \mathrm{Watts} / \mathrm{m}^{2} \\
&=4 \times 10^{10} \mathrm{Watts} / \mathrm{m}^{2} / \mathrm{cm}^{-1}
\end{aligned}
$$

Therefore the electric field generated is given by:

$$
\begin{aligned}
E & =\left(\frac{4 \times 10^{10}}{1.33 \times 10^{-3}}\right)^{\frac{1}{2}} \text { Volts } / \mathrm{m} \\
& =5.5 \times 10^{8} \text { Volts } / \text { meter }
\end{aligned}
$$

We further note, however, as has been pointed out by Al Seivers[14], that if one is using this field to drive a specific transition of narrow bandwidth, then one has to take account of this and the effective field will be lower, and for instance for a $1 \mathrm{~cm}^{-1}$ bandwidth, it will be lower by a factor of about 100 .

Magnetic fields are given by E/c, and therefore are of the order of 1 Tesla for this beamline. Finally we would like to point out that if the bunch charge were to be increased to 1 nanoCoulomb from our 135 picoCoulombs, then the electric field would increase by $(1000 / 135)^{2}$, or 64 , making a magnetic field of $64 \mathrm{~T}$. Such bunch charges have already been realized both at the Stanford Linear Accelerator Center and the source development facility at Brookhaven, albeit at very low repetition rates of $100 \mathrm{~Hz}$ or less compared to our $75 \mathrm{MHz}$. 


\section{CONCLUSIONS}

We have constructed a beamline capable of delivering 100 Watts of broadband $\mathrm{THz}$ light in the range $0.1-10 \mathrm{THz}$ into a user laboratory. The beamline is based on our groundbreaking $4^{\text {th }}$. generation light source, and calculations using a newly developed code which takes account of multiparticle effects as well as near-field effects and which we have benchmarked against an earlier accelerator.

\section{ACKNOWLEDGEMENTS}

This work was supported by U.S. DOE Contract No. DE-AC05-84-ER40150, the Office of Naval Research, the Air Force Research Laboratory, the Army Night Vision Laboratory, the Commonwealth of Virginia and the Laser Processing Consortium.

\section{REFERENCES.}

1. G.L. Carr, M.C. Martin, W.R. McKinney, K. Jordan, G.R. Neil and G.P. Williams, Nature 420, 153-156 (2002).

2. G.P. Williams, Phil Trans. R. Soc. Lond. A 362, 403 (2004).

3. G.R. Neil, et al., Phys. Rev. Lett. 84, 662 (2000).

4. D.K. Campbell, S. Flach and Y.S. Kivshar, Physics Today, January 2004, p 43.

5. D. H. Auston, K. P. Cheung, J. A. Valdmanis, and D. A. Kleinman, Phys. Rev. Letts. 53, 1555 (1984).

6. J.D. Jackson, Classical Electrodynamics, Wiley, New York 1975

7. G. Neil et al., these proceedings.

8. R. A. Bosch, Review of Scientific Instruments 73, 1423 (2002).

9. Y.-L. Mathis, B. Gasharova and D. Moss, J. Bio. Phys. 29, 313 (2003).

10. O. Chubar, P. Elleaume, "Accurate And Efficient Computation Of Synchrotron Radiation In The Near Field Region", proc. of the EPAC98 Conference, 22-26 June 1998, p.1177-1179.

11. W.D. Duncan and G.P. Williams, Applied Optics 22, 2914 (1983).

12. R. M. S. Lobo, J. D. Laveigne, D. H. Reitze, D. B. Tanner and G. L. Carr, Review of Scientific Instruments, 73, 1 (2002).

13. G. Lüpke, N. H. Tolk, L. C. Feldman, J. Appl. Phys. 93, 23162003.

14. A. J. Sievers, private communication. 